\title{
Cerebral Palsy: Enhancing Movement Opportunity with Help from the Care Team
}

\author{
Carrah Nelson a, Kirsten Fuchs ${ }^{\text {a, }}$ Lacie W. Pennington b, \& Colin G. Pennington a,* \\ a Tarleton State University, Stephenville, TX 76401, United States, \\ b Sport Therapy, Fort Worth, TX76401, United States. \\ *Corresponding author Tel: +55(35) 3829-5132; Email: cpennington@tarleton.edu \\ DOI: https://doi.org/10.34256/ijpefs2043
}

Received: 12-09-2020, Revised: 20-10-2020; Accepted: 20-10-2020; Published: 22-10-2020

Abstract: Cerebral palsy is the most common motor disability in childhood. Cerebral palsy can significantly impact the amount of physical activity an individual obtains, and the scope and quality of the physical activity may be limited by cerebral palsy. High-quality physical education can integrate children with cerebral palsy into movement programing, as to enable children with cerebral palsy to achieve goals related to cognitive skills associated with exploring physical health, maintaining physical health, as well as affective values towards physical health, movement, play, and general wellbeing. This article provides a basic overview of the physical characteristics of cerebral palsy, as well as outlines ways the child's educational care-team can work with physical educators and physical therapist to enhance movement skills and health behavior.

Keywords: Cerebral palsy, Physical education, Special education, Physical therapy, Adapted physical education, Inclusion

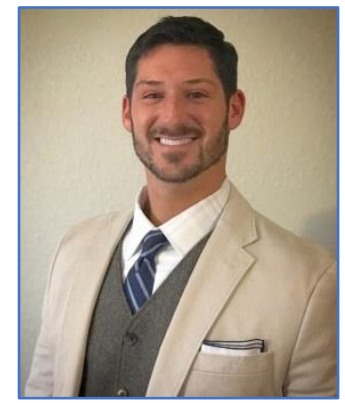

Colin G. Pennington Ph.D is an Assistant Professor of Kinesiology at Tarleton State University (USA) where he works with Exercise and Sport Study majors, and conducts research on physical education teacher effectiveness and other health-related applications of the kinesiology sub-disciplines. He currently teaches courses including coaching and sport leadership, adaptive physical education, physiology of exercise, anatomical kinesiology, and others. His interests and research focus on teacher socialization, physical education teacher training, character development programs within physical education and sport, and health and wellness.

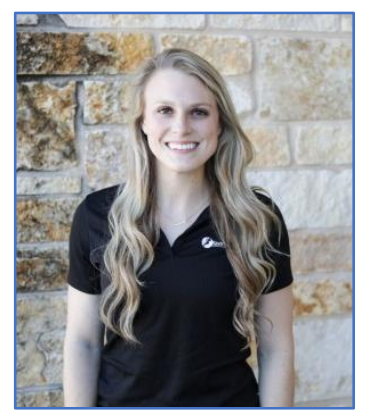

Lacie $\mathbf{w}$. Pennington is a physical therapist in Fort Worth, Texas. Lacie has a variety of clinical experiences including post-operative orthopedic surgery rehabilitation, acute care in cardiac intensive care unit, sports medicine rehabilitation, and rehabilitation to individuals with neurological impairments. She has served as volunteer, technician, and student physical therapist in numerous cities, and has experience working with patients possessing a wide-range of functional abilities. The majority of Lacie's clinical interests have focused on sport-related injuries, and return-to-sport and injury preventative programs. Her mission is to live out the message in 1 Peter 4:10, "Each of you should use whatever gift you have received to serve others, as faithful stewards of God's grace in its various forms."

Kirsten L. Fuchs is a senior at Tarleton State University. She is currently pursuing her degree in Kinesiology with plans to attend Park University to get her Doctorate of Chiropractic. She plans to eventually work as a Sport Chiropractor.

Carrah Nelson is a senior at Tarleton State University. She is currently pursuing her degree in Kinesiology. 


\section{Introduction}

Cerebral palsy is the result of a brain injury or abnormality. Cerebral palsy is the most common motor disability in childhood, and there are several classifications for this disability [1]. Some classifications include monoplegia, where any one part of the body is involved, hemiplegia, where one complete side of the body is involved, or quadriplegia, where the total body is involved. There are also associated dysfunctions that can accompany cerebral palsy: mental retardation, speech problems, seizures, or reflex problems [2]. The most common type of cerebral palsy is spastic, which is damage to the cerebrum and is characterized by abnormal muscle tightness and stiffness and shortened Achilles tendon and scissoring gait (1). A key characteristic of cerebral palsy is that every case is completely individual with differing variations of health concerns (2). Cerebral palsy is categorized in five levels (1):

I. Ability to walk without limitations

II. Ability to walk except for long distances and poor balance

III. Needs adaptive equipment to walk

IV. Independence of mobility with the need of powered mobility assistance or wheelchair

V. Extensive physical support with the use of assisted technology

Cerebral palsy is a complicated condition with multiple causes; multiple clinical types; and multiple developmental pathologies, such as intellectual disability, autism, epilepsy, and visual impairment [3]. These various features of the condition result in a nonspecific and nonprogressive disorder of posture and movement control. Besides cognitive abilities being frequently affected, accompanying symptoms also include visible motor impairments [4, 5]. Considerable effort has recently been made to improve the diagnosis of cerebral palsy in childhood and to establish early intervention aiming to improve functional movement outcomes [5].

\section{The Potential Impact of Physical Education Programs}

Understanding how the physical characteristics of the disability helps explain why physical education is so important to those affected by cerebral palsy. Organizations such as the National Institutes of Health (NIH) and the American College of Sport Medicine
(ACSM) recommends 30 minutes of moderate to intense physical exercise from two to four times a week for most individuals [6]. Individuals with cerebral palsy very rarely meet this guideline. Cerebral palsy patients are already at risk for heart and flexibility issues, so not achieving the minimum NIH and ACSM threshold for physical activity compounds physical health concerns. Inclusion in a high-quality $\mathrm{K}-12$ physical education program can give students with cerebral palsy the opportunity to not only improve their physical health quality but social, emotional, and cognitive health as well. Improvement in all holistic areas of personal health benefits the individual most when physical activity is introduced at a young age, as health and physical activity patterns developed in childhood tend to perpetuate into adulthood [7]. Quality physical education includes more progressive and holistic teaching opportunities than offed by playing dodgeball, for example. A high-quality physical educator establishes a learning environment which enhances the educational experiences and opportunities to educate the 'whole' child [i.e. cognitive, emotional, social components of physical activity and health] on body and muscle control as well as even critical thinking [8]. Students with cerebral palsy should not be denied the opportunities highquality physical education provides.

\section{The Potential Impact of Physical Therapy}

Physical therapy plays a major role for individuals with cerebral palsy early in life [9]. Highly individualized physical therapy can enhance the overall quality of life for a person with cerebral palsy. Some key goals of physical therapy would to be build independence while encouraging a healthy lifestyle. For individuals with cerebral palsy, physical therapy is more than just meeting with a therapist a couple of time a week for an hour. What may appear to simply be a physical therapist using a yoga ball to enhance reflexes, could actually be the steppingstone to an occupational therapist improving fine and gross motor control. Improved fine and gross motor control then makes it possible for to the individual with cerebral palsy to handle smaller objects such as utensils. Such an achievement could lead to the individual being able to eventually write, color, or even hold a spoon to help feed themselves rather than someone else doing it for them [i.e. greater independence].

Physical therapy has been shown to improve muscle strength, muscular endurance and joint range 
of movement in children with cerebral palsy [10]. Successful physical therapy may include passive gentle range of motion exercises and stretches across major joints whereby increased range-of-motion results. In addition, muscle strength is achieved by performing regularly scheduled progressively increasing resistive exercises involving all major muscle groups; specific physiotherapy exercises are designed to improve balance, postural control, gait, and assist with mobility and transfers (for example from bed to wheelchair [10].

Functional strength training combined with plyometric exercises and balance training have been used to improve function in individuals with cerebral palsy [10]. Plyometric exercise improves muscle power, which includes strength and speed; in regards to functional strength training, targeting specific muscles is most effective in muscle activation.

\section{Tips and Suggestions for Improving Physical Capabilities}

Physical educators and educational support staff should encourage students with cerebral palsy to be physically active and recommend exercise testing when the child experiences limitations in activities because of physical exhaustion [11]. An Admission, Review, and Dismissal (ARD) meeting is a yearly assessment, whereby participants have the opportunity to ask questions and provide input regarding the development of the child [12]. The ARD team includes teachers, parents, therapist, and some school administration. Importantly, this meeting must include the physical education teacher. At the time of an ARD meeting, the entire care team will develop an Individualized Educational Plan (IEP) for the student. An IEP represents goals the care team develops for the student to accomplish in the next academic year. The IEP provides a blueprint moving forward for the physical education teacher develop inclusive lesson plans for the benefit the child. The IEP will also guide the physical education teacher on what is appropriate movement based on the individual child's abilities and risks.

As indicated by Verschuren and colleagues (2006), children with cerebral palsy are often raised in an environment where physical activity primarily occurs through formal physical therapy sessions - it is rare for children to be fully included in traditional physical education and organized sports events for children with disabilities [although that is an ultimate goal that is slowly being achieved] [11]. An example of appropriate goals for an individual with cerebral palsy include, not just the psychomotor aspects of physical education, but also the cognitive skills associated with exploring physical health, maintaining physical health [13], as well as developing affective values towards physical health, movement, play, and general wellbeing. Children with cerebral palsy often experience physical activity exclusively from interactions with health professionals that are generally related to daily function, increased muscle tone, and decreased range of motion; these are important concerns, especially during the developmental years, but there is often little discussion of healthy lifestyles that involve physical activity and sedentary behavior reduction [11]. This is an area where the physical education staff could integrate their expertise into the physical health aspect of the student's IEP. Promoting elements of goal setting, sportsmanship [14], and cooperation [15] as components of a holistic physical education program, could provide a means to meeting the challenge of promoting life-long movement and enjoyment of physical activity and sporting experiences.

A further suggestion is for the ARD care team to develop a close working relationship with a physical therapist. The physical therapist can offer assistance as to what psychomotor patterns relating to muscular strength, muscular endurance, flexibility, and physical mechanics need to be improved upon and what is already mastered. A working partnership between physical therapy and physical education can result in the greater good for the student [9]. Each time a cerebral palsy patient improves motor growth in one foundational movement pattern, a bridge is built to another potential movement skill; in this sense, scaffolding of progressive moments is achieved. The lasting result could be the student being able transition from a wheelchair to walking with adaptive equipment, or being able to write their own name.

\section{Conclusion}

Physical education can improve body function, which then leads to better muscle movements, which increases flexibility, leading to improved total body control. When a person with cerebral palsy accomplishes these or similar goals, the result is enhanced independence and self-esteem. Research suggests that improved independence, self-esteem, and self-efficacy pushes individuals to keep breaking down walls and meeting new goals aligned with possessing a healthy physical culture. This chain 
reaction of positive outcomes starts with high quality physical education for all. Because cerebral palsy is associated with multiple associated and secondary medical conditions, its management requires a multidisciplinary team approach. It is important to remember that most children with cerebral palsy grow up to be productive adults.

\section{References}

[1] C.L. Richards, \& F. Malouin, (2013), Cerebral palsy: definition, assessment and rehabilitation, In Handbook of Clinical Neurology (Vol. 111, pp. 183-195). Elsevier.

[2] K. Vitrikas, H. Dalton, \& D. Breish, Cerebral palsy: An overview, American Family Physician, 101(4) (2020) 213-220.

[3] A.H. MacLennan, S.C. Thompson, \& J. Gecz, Cerebral palsy: causes, pathways, and the role of genetic variants, American Journal of Obstetrics and Gynecology, 213(6) (2015) 779-788. https://doi.org/10.1016/j.ajog.2015.05.034

[4] S.S. Philip, A. Guzzetta, O. Chorna, G. Gole, \& R.N. Boyd, Relationship between brain structure and Cerebral Visual Impairment in children with Cerebral Palsy: A systematic review, Research in Developmental Disabilities, 99 (2020) 103580. https://doi.org/10.1016/j.ridd.2020.103580

[5] J. Fluss, \& K. Lidzba, Cognitive and academic profiles in children with cerebral palsy: a narrative review, Annals of Physical and Rehabilitation Medicine, Accpeted (2020). https://doi.org/10.1016/j.rehab.2020.01.005

[6] W.L. Haskell, I.M. Lee, R.R. Pate, K.E. Powell, S.N. Blair, B.A. Franklin, C.A. Macera, G.W. Heath, P.D. Thompson, \& A. Bauman, Physical activity and public health: updated recommendation for adults from the American College of Sports Medicine and the American Heart Association, Circulation, 116(9) (2007) 1081.

https://doi.org/10.1161/CIRCULATIONAHA.107.1 85649

[7] C.G. Pennington, Sport Education and Physical Activity: Recommendations for Maximizing the Model, International Journal of Physical Education, Fitness and Sports, 8(1) (2019a) 122125. https://doi.org/10.26524/ijpefs19114.

[8] C.G. Pennington, Faith, Physical Activity, and Physical Education, The Canadian Journal for Scholarship and the Christian Faith. Special Issue: Exploring the Relationship Between Physical Activity and the Christina Faith, (1) (2019b) 2-9.

[9] D.L. Damiano, Activity, activity, activity: rethinking our physical therapy approach to cerebral palsy, Physical Therapy, 86(11) (2006) 1534-1540.

https://doi.org/10.2522/ptj.20050397
[10] D.R. Patel, M. Neelakantan, K. Pandher, \& J. Merrick, Cerebral palsy in children: a clinical overview, Translational Pediatrics, 9(Suppl 1) (2020) S125.

https://doi.org/10.21037/tp.2020.01.01

[11] O. Verschuren, M.D. Peterson, A.C. Balemans, \& E.A. Hurvitz, Exercise and physical activity recommendations for people with cerebral palsy, Developmental Medicine \& Child Neurology, 58(8) (2016) 798-808.

[12] K.L. Carroll, J. Leiser, \& T.S. Paisley, Cerebral palsy: physical activity and sport, Current Sports Medicine Reports, 5(6) (2006) 319-322. https://doi.org/10.1007/s11932-006-0060-x

[13] C.G. Pennington, (2019c). Promoting Youth Physical Activity Using the Transtheoretical Model of Behavioral Change, Preceding's for the International Conference on Health, Nutrition, Exercise Science. A1.

[14] C.G. Pennington, Moral Development and Sportsmanship in Interscholastic Sports and Physical Education, Journal of Physical Education, Recreation \& Dance, 88(9) (2017) 3642.

https://doi.org/10.1080/07303084.2017.1367745

[15] C.G. Pennington, Creating and Confirming a Positive Sporting Climate, Journal of Physical Education, Recreation \& Dance. 90(4) (2018) 1520.

https://doi.org/10.1080/07303084.2019.1568936

Acknowledgement

NIL

Funding

This study was not funded by any grant

\section{Ethics Approval}

This study doesn't require ethical approval

\section{Authors Contribution}

Methodology, manuscript preparation, review and editing ; Carrah Nelson, Manuscript preparation, review and editing; Kirsten Fuchs, Manuscript preparation, review and editing; Lacie W. Pennington; Conceptualization, Supervision, Manuscript preparation, review and editing; Colin G. Pennington. All the authors have read and approved the manuscript.

\section{Conflict of interest}

The authors declare that they have no actual or potential conflict of interest, including financial, personal or other relationships with people or organizations that could have inappropriately influenced this work.

\section{About The License}

(C) The author(s) 2020. The text of this article is open access and licensed under a Creative Commons Attribution 4.0 International License 